\title{
Alteraciones del metabolismo de la glucosa en donante y receptor
}

\section{Alterations of glucose metabolism in donor and recipient}

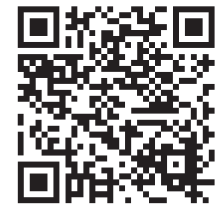

\author{
Itzel Anahí Martínez-Juárez*
}

* Área de Nefrología. Departamento de Medicina Interna. Hospital General «Dr. Manuel Gea González».

\section{EVALUACIÓN DEL CANDIDATO A DONADOR RENAL}

\section{Recomendaciones}

- No podrán ser donadores renales los pacientes con diagnóstico de diabetes mellitus (DM) 10 DM2. (Obligatorio) $1^{1-5}$

- Todos los potenciales donadores mexicanos deberán someterse a curva de tolerancia oral a la glucosa (CTOG) de 75 g. (Obligatorio). ${ }^{3,6,7}$

- Pacientes mayores de 60 años con CTOG alterada como único factor de riesgo podrán ser candidatos a donar sólo en casos excepcionales (Deseable) ${ }^{7,8}$

- Los donadores con CTOG alterada o hemoglobina glucosilada (HbA1c) alterada ${ }^{9,10}$ deberán ser informados del riesgo de progresión a diabetes (10-30\% a los 5 años) y las complicaciones renales a largo plazo. (Obligatorio)..$^{8,11-15}$

- Podrán utilizarse calculadoras de riesgo de progresión de DM2 con fines informativos al donador como: FINDRISC (Finnish Diabetes Risk Score), ${ }^{16}$ e índices de sensibilidad a la insulina como Matsuda index, HOMA-IR y HOMA2S. (Obligatorio). ${ }^{17}$

\section{Sustento Científico}

De los 415 millones de personas diagnosticadas con DM2, $80 \%$ habitan en países de bajos recursos.
México tiene 11.5 millones de habitantes con DM2 y se encuentra dentro de los primeros 10 países con mayor número de casos. ${ }^{10}$

Los donadores vivos tienen seis veces más riesgo de ERC (enfermedad renal crónica) a 15 años en comparación con población sana, pero el riesgo acumulado se mantiene por debajo de $1 \%$ (riesgo absoluto de ERC a lo largo de su vida es 14 casos por 10,000 habitantes en población sana, 90 casos por 10,000 habitantes en donadores renales y 326 casos por 10,000 habitantes en población general). ${ }^{18,19}$

La incidencia de diabetes postnefrectomía ha sido estudiada en diferentes grupos étnicos, se ha descubierto que la comunidad hispano latina tiene 2.5 más riesgo de desarrollar diabetes posterior a la donación renal. ${ }^{20}$

En un estudio mexicano se demostró que $30 \%$ de los pacientes con diabetes mantenían $\mathrm{HbA} 1 \mathrm{c}$ mayores de 10, en comparación con las estadísticas del primer mundo, donde apenas $5 \%$ de la población muestra esos niveles, ${ }^{21}$ lo cual probablemente sea debido a la falta de seguimiento estrecho y falta de recursos económicos de un país en vías de desarrollo, donde la demanda es mayor que los servicios sanitarios disponibles.

Las guías KDIGO 2017 y las guías europeas mencionan que la decisión de aceptar un donador prediabético o diabético deberá ser individualizada, informando los riesgos existentes a largo plazo en el pronóstico de la enfermedad. ${ }^{8}$ Sin embargo, en México no se podrá aceptar a donadores diabéticos, dada la alta predisposición genética, prevalencia de obesidad 
y sobrepeso y por ende, la tórpida evolución reportada en nuestra comunidad latina. ${ }^{12}$

Los pacientes donadores diabéticos pueden encontrarse en estadios iniciales de hiperfiltración y acelerar la progresión de la enfermedad renal al ser nefrectomizados. ${ }^{22}$ Los pacientes nefrectomizados pierden de $25-40 \%$ de la TFG (tasa de filtración glomerular), ${ }^{23}$ lo cual en pacientes diabéticos podría ocasionar rápido deterioro de la función renal descrito a partir del estadio G3a hasta de $9 \mathrm{~mL} / \mathrm{min} / 1.73$ por año. ${ }^{24} \mathrm{La}$ ausencia de microalbuminuria no asegura la ausencia de daño renal por diabetes, ya que $40 \%$ de los pacientes sin microalbuminuria pueden encontrarse en estadios avanzados de enfermedad renal. ${ }^{25}$

El donador deberá entender las implicaciones de donación a corto y largo plazo. El centro hospitalario deberá consensar el límite de riesgo aceptable postdonación según las características físicas y demográficas del donador. Se cuenta con la herramienta ESRD Risk Tool for Kidney Donor Candidates, la cual calcula el riesgo de ERC a 15 años según características del donador. ${ }^{1}$

\section{EVALUACIÓN Y MANEJO DEL RECEPTOR DE TRASPLANTE RENAL}

\section{Recomendaciones}

\section{1) Selección de receptores renales con DM}

- Los pacientes diabéticos tipo 1 y 2 deberán ser candidatos al TR siempre y cuando tengan una esperanza de vida mayor de cinco años según sus comorbilidades y la apreciación clínica. (Obligatorio). ${ }^{26}$

- Pacientes diabéticos de > 10 años de evolución deberán tener documentado el índice tobillo brazo, en caso de resultar $<0.9-1.3>$ se deberá solicitar Doppler arterial y valoración por cirugía vascular. (Deseable). ${ }^{27-29}$

- Solicitar placa simple de pelvis para valorar grado de calcificación vascular y en caso de ser moderada o grave, se solicitará TAC y valoración por cirujano de trasplantes previo al procedimiento. (Deseable). ${ }^{26,30}$

- Lo ideal sería que todos los pacientes fueran valorados por cirugía vascular y dermatología previo a la realización del trasplante. (Deseable). ${ }^{28,30}$

2) Diagnóstico de diabetes mellitus de inicio posterior al trasplante
- El diagnóstico de DMPT deberá realizarse a partir del tercer mes. (Obligatorio). ${ }^{31}$

- El diagnóstico de DMPT es exactamente igual al diagnóstico de diabetes mellitus descrito en las guías de la American Diabetes Association (ADA). (Obligatorio). ${ }^{31}$

- Medir CTOG en el primer año postrasplante y/o $\mathrm{HbA1c}$ si los niveles de $\mathrm{Hb}$ se encuentran estables y con ausencia de anemia. (Obligatorio). ${ }^{32}$

- El tratamiento inicial podrá ser con insulina, posteriormente hipoglucemiantes orales y cambios en el estilo de vida. (Obligatorio). ${ }^{26}$

3) Inmunosupresión en receptores de trasplante renal con DM

- No deberán hacerse cambios en el esquema de inducción de acuerdo al perfil glucémico del paciente. (Obligatorio).

- No deberán hacerse cambios en el esquema o mantenimiento con inhibidores de calcineurina de acuerdo con perfil glucémico, éste será normado sólo por el perfil inmunológico del paciente. (Obligatorio).

- Monitorizar por alto riesgo de rechazo celular, aunque la literatura es controversial. (Sugerido).

- No recomendamos esquemas libres de esteroides. Sin embargo, los pacientes DM1 y DM2 podrán ser candidatos a esquemas de reducción rápida de esteroides siempre y cuando sean de bajo riesgo inmunológico. (Obligatorio).

\section{Sustento Científico}

\section{1) Selección de receptores renales con DM}

Cincuenta por ciento de los pacientes que inician terapia de sustitución renal tienen ERC secundaria a DM2, a pesar de esto en la mayoría de las series descritas apenas $20 \%$ de los receptores de trasplante renal tiene DM2 y 14\% DM1, lo que describe menos probabilidades de recibir un trasplante en esta población. Las tasas de supervivencia en el postrasplante son similares en pacientes diabéticos y no diabéticos siempre y cuando se mantengan con injerto funcional, no hay diferencia en la supervivencia del injerto entre estos grupos. ${ }^{33}$ Aunque existe literatura previa con resultados controversiales. ${ }^{34}$

En 2003 el término NODAT (New Onset Diabetes Associated Transplant) fue introducido por primera 
vez; sin embargo, debido a la falta de escrutinio no se podía asegurar que todos los pacientes iniciaran con el problema posterior al trasplante, por lo que en 2014 el término es modificado a DMPT (Diabetes Mellitus Post-Transplant). ${ }^{35}$ Entre 5 y $30 \%$ de los pacientes trasplantados desarrollarán DMPT en el primer año postrasplante. ${ }^{31}$ En una población japonesa se vinculó al Homeostasis Model Assessment of Insulin Resistance como un buen predictor de riesgo. ${ }^{36}$

La incidencia acumulada de amputaciones incrementa a $18.7 \%, 37.5 \%, 43.8 \%$ y $50 \%$ en el primer, segundo, tercer y cuarto año de inicio de diálisis respectivamente, por lo que el escrutinio de enfermedad vascular periférica será un requerimiento en este tipo de pacientes. ${ }^{37}$ La valoración integral de las extremidades es ideal, por lo que se sugiere la visita previa al trasplante de un dermatólogo, cirujano vascular y ortopedista.

2) Diagnóstico de diabetes mellitus de inicio posterior al trasplante

El diagnóstico de DMPT deberá realizarse una vez que los inmunosupresores tengan una dosificación estable y con dosis baja de esteroides, lo cual ocurre a partir del tercer mes.

El diagnóstico de DMPT es exactamente igual al diagnóstico de diabetes mellitus descrito en las guías de la American Diabetes Association (ADA). ${ }^{31}$

- Glucosa en ayuno > 126 mg/dL en más de dos ocasiones.

- Glucosa al azar > 200 mg/dL más síntomas.

- CTOG con $75 \mathrm{~g}$ con medición > $200 \mathrm{mg}$ a las dos horas.

- $\mathrm{HbA1c}>6.5 \%$.
Se preferirá medir CTOG en el primer año, ya que la fisiopatología de la PTDM se basa en una disfunción de la célula $B$ pancreática, por lo que la glucosa de ayuno alterada puede no ser un buen marcador, puesto que su valor es dependiente del funcionamiento hepático más que de una secreción inapropiada de insulina como sucede en las anormalidades de una curva de tolerancia a la glucosa. ${ }^{32}$ La HbA1c se estabilizará entre los tres meses y al año postrasplante, por lo que se sugiere tomarla como marcador de PTDM posterior a este intervalo. ${ }^{26}$

En la siguiente Tabla 1 se esquematizan los algoritmos temporales para determinar un diagnóstico de diabetes mellitus postrasplante y su manejo. ${ }^{26}$ 3) Inmunosupresión en receptores de trasplante renal
con DM

No deberán hacerse cambios en el esquema de inducción de acuerdo al perfil glucémico del paciente, ya que la evidencia existente es de bajo poder estadístico.

En inducción alemtuzumab (anti-CD52) se demostró tendencia a menor riesgo de PTDM y menor riesgo de rechazo agudo probado por biopsia en comparación con basiliximab (anti-IL-2R), pero no contra timoglobulina (antitimocito). ${ }^{37}$

No deberán hacerse cambios en el esquema de mantenimiento con inhibidores de calcineurina de acuerdo con perfil glucémico, éste será normado sólo por el perfil inmunológico del paciente.

Existen artículos que sugieren un incremento en la mortalidad de los pacientes que desarrollan PTDM; sin embargo, el rechazo impacta de manera estadísticamente significativa en la pérdida del injerto, por lo que

Tabla 1: Diagnóstico y tratamiento de Diabetes Mellitus de nuevo inicio postrasplante.

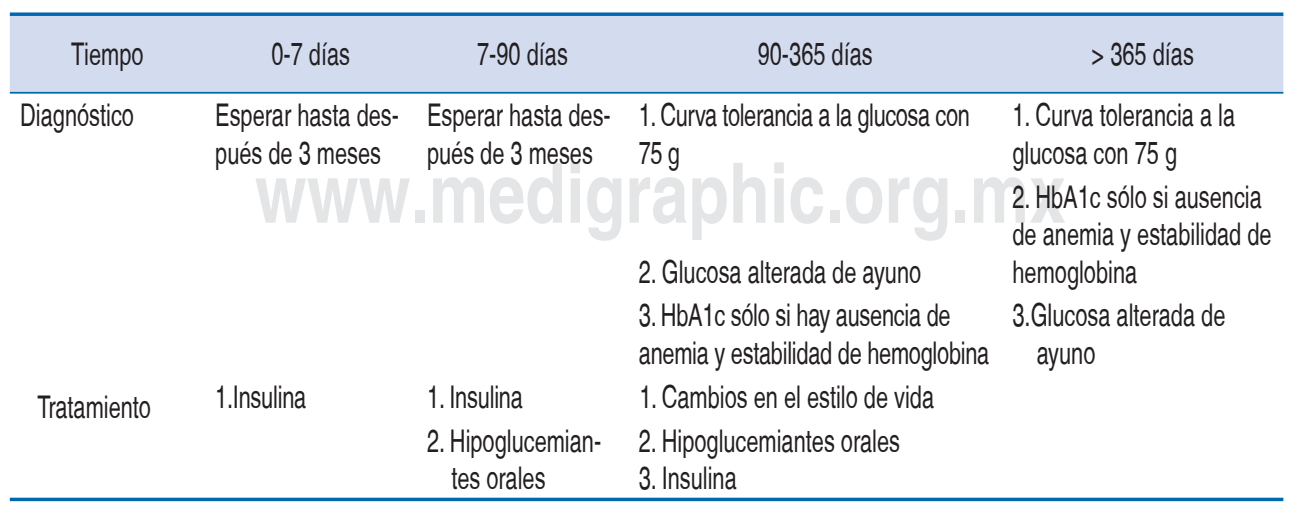


el cambio de inmunosupresión deberá ser regido por factores como el riesgo inmunológico del paciente. ${ }^{34,38,39}$

Existe evidencia limitada que sustente el cambio de tacrolimus a ciclosporina o esquemas ahorradores de inhibidores de calcineurina, ${ }^{40}$ así como como adición de belatacept o tofacitinib. ${ }^{41} \mathrm{La}$ evidencia para eventos negativos relacionados al cambio por sirolimus está bien descrita. ${ }^{42}$

Los artículos vinculan un incremento de riesgo de rechazo predominantemente celular en pacientes con DM pre o PTDM ${ }^{43,44}$ y no se sabe si es una asociación bilateral, es decir, que más rechazo conlleve a incremento de esteroides o inversamente más DM2 o riesgo de DMPT predispone a esquemas de reducción rápida de esteroides que incrementan el riesgo de rechazo. ${ }^{34,38,39}$ La literatura es controversial.

Los pacientes DM1 y DM2 podrán ser candidatos a esquemas de reducción rápida de esteroides (pero no de esquemas libres de esteroides), siempre y cuando sean de bajo riesgo inmunológico definido por:

- Panel reactivo de anticuerpos $<20 \%$

- Ausencia de anticuerpos anti-HLA dirigidos contra el donante

- Isquemia fría < 30 horas

- Sin infección por el virus de la inmunodeficiencia humana $(\mathrm{VIH})$

Cuando los rechazos agudos ocurren dentro de los tres primeros meses del trasplante, no tienen un efecto en falla del injerto censurada a muerte o nefropatía crónica del injerto. Los receptores que tienen su primer rechazo después del primer año postrasplante mostraron un efecto negativo en supervivencia global y del injerto. ${ }^{45}$ Por tal motivo, un rechazo agudo en un paciente con esquema de reducción rápida de esteroides probablemente será tratado con éxito sólo con la adición de esteroides.

Existen estudios que han comparado el retiro temprano de esteroides contra el uso crónico en dosis bajas es pacientes con bajo riesgo inmunológico a cinco años de seguimiento observando desenlaces de supervivencia global (5.8 vs. $6.7 \%$ ) y del injerto (5.8 vs. 3.6) similares así como rechazos moderados y graves (7.9 vs. 6.2) de éstos, el grupo de corticoesteroides tuvo mayor requerimiento de timoglobulina que los de retiro rápido de esteroides (65 vs. 27); sin embargo, sí hubo mayor incidencia de rechazos leves BANFF $1 \mathrm{~A}$ en el grupo de retiro rápido de esteroides (17.8 vs. $10.8 p=0.05)$, los cuales respondieron adecuadamente a MTPDN $7 \mathrm{mg} / \mathrm{kg}$ en tres dosis con posterior esquema de reducción. El estudio manejó los niveles de tacrolimus deseados entre 10-20 primer mes y 5-10 en los primeros 90 días. El micofenolato fue suministrado en los primeros 15 días con dosis de $3 \mathrm{~g} /$ día y $70 \%$ de los pacientes para cada grupo utilizó timoglobulina. No existen beneficios claros de un esquema de reducción de esteroides. ${ }^{46}$ Este último estudio fue extendido hasta 15 años sin mostrar diferencia en supervivencia del injerto y supervivencia del paciente. ${ }^{47}$

La revisión sistemática de Pascual y Galeano describe el retiro de esteroides entre el tercero y sexto mes con desenlaces a los tres años sin cambios en supervivencia global, ni supervivencia del injerto ni rechazos, siempre y cuando la inmunosupresión se mantenga con MMF y tacrolimus. ${ }^{48}$

\section{REFERENCIAS}

1. Grams ME, Sang Y, Levey AS et al. Kidney-failure risk projection for the living kidney-donor candidate. N Engl J Med. 2016; 374: 411-421.

2. Spanish Society of Nephrology (SEN) and Spanish Transplant Organization (ONT). Recommendations for living-donor kidney transplantation. Nefrologia. 2010; 30: 1-105.

3. Boudville N, Isbel N. The CARI guidelines. Donors at risk: impaired glucose tolerance. Nephrology (Carlton). 2010; 15 (Suppl 1): S133-S136.

4. Andrews PA, Burnapp L, Manas D et al. Summary of the British Transplantation Society/Renal Association U.K. Guidelines for living donor kidney transplantation. Transplantation. 2012; 93: 666-673.

5. Dudley C, Harden P. Renal Association Clinical Practice Guideline on the assessment of the potential kidney transplant recipient. Nephron Clin Pract. 2011; 118: s209-s224.

6. Morris RD, Rimm DL, Hartz AJ et al. Obesity and heredity in the etiology of non-insulin-dependent diabetes mellitus in 32662 adult white women. Am J Epidemiol. 1989; 130: 112-122.

7. Lentine $\mathrm{KL}$, Kasiske $\mathrm{BL}$, Levey $\mathrm{AS}$ et al. KDIGO Clinical Practice Guideline on the Evaluation and Care of Living Kidney Donors. Transplantation. 2017; 101 (8S Suppl 1): S1-S109.

8. Pascual J, Abramowicz D, Cochat $P$ et al. European renal best practice guideline on the management and evaluation of the kidney donor and recipient. Nefrologia. 2014; 34: 293-301.

9. Nathan DM, Davidson MB, DeFronzo RA, Heine RJ, Henry $\mathrm{RR}$, Pratley $\mathrm{R}$ et al. Impaired fasting glucose and impaired glucose tolerance: implications for care. Diabetes Care. 2007; 30: 753-759.

10. Bello-Chavolla OY, Rojas-Martinez R, Aguilar-Salinas CA, Hernández-Avila M. Epidemiology of diabetes mellitus in Mexico. Nutr Rev. 2017; 75 (suppl 1): 4-12.

11. Wareham NJ, Byrne CD, Williams R et al. Fasting proinsulin concentrations predict the development of type 2 diabetes. Diabetes Care. 1999; 22: 262-270.

12. Vigneault CB, Asch WS, DahI NK, Bia MJ. Should living kidney donor candidates with impaired fasting glucose donate? Clin J Am Soc Nephrol. 2011; 6 (8): 2054-2059.

13. Okamoto $\mathrm{M}$, Suzuki $\mathrm{T}$, Fujiki $\mathrm{M}$ et al. The consequences for live kidney donors with preexisting glucose intolerance 
without diabetic complication: analysis at a single Japanese center. Transplantation. 2010; 89: 1391-1395.

14. Shinzato $T$, Kurosawa $A$, Kubo $T$ et al. No significant differences in short-term renal prognosis between living kidney donors with and without diabetes. Clin Exp Nephrol. 2018; 22 (3): 694-701.

15. Feig DS, Zinman B, Wang X, Hux JE. Risk of development of diabetes mellitus after diagnosis of gestational diabetes. CMAJ. 2008; 179 (3): 229-234.

16. Lindstrom J, Tuomilehto J. The diabetes risk score: a practical tool to predict type 2 diabetes risk. Diabetes Care. 2003; 26: 725-731.

17. Lorenzo C, Hazuda HP, Haffner SM. Insulin resistance and excess risk of diabetes in Mexican-Americans: the San Antonio Heart Study. J Clin Endocrinol Metab. 2012; 97 (3): 793-799.

18. Lam NN, Lentine KL, Levey AS, Kasiske BL, Garg AX. Longterm medical risks to the living kidney donor. Nat Rev Nephrol. 2015; 11 (7): 411-419.

19. Muzaale $A D$, Massie $A B$, Wang $M C$ et al. Risk of end-stage renal dis- ease following live kidney donation. JAMA. 2014; 311: 579-586.

20. Holscher CM, Bae S, Thomas AG et al. Early hypertension and diabetes after living kidney donation: a national cohort study. Transplantation. 2019; 103 (6): 1216-1223.

21. Alegre-Díaz J, Herrington W, López-Cervantes $M$ et al. Diabetes and cause-specific mortality in Mexico City. N Engl J Med. 2016; 375 (20): 1961-1971.

22. Lopes GS, Lemos CC, Mandarim-De-Lacerda CA, Bregman R. Effect of unilateral nephrectomy on renal function of diabetic rats. Histol Histopathol. 2004; 19: 1085-1088.

23. Garg AX, Muirhead N, Knoll G et al. Proteinuria and reduced kidney function in living kidney donors: a systematic review, meta-analysis, and meta-regression. Kidney Int. 2006; 70: 1801-1810.

24. Iwai T, Miyazaki M, Yamada $\mathrm{G}$ et al. Diabetes mellitus as a cause or comorbidity of chronic kidney disease and its outcomes: the Gonryo study. Clin Exp Nephrol. 2018; 22 (2): 328-336.

25. Alicic RZ, Rooney MT, Tuttle KR. Diabetic Kidney Disease: challenges, progress, and possibilities. Clin J Am Soc Nephrol. 2017; 12 (12): 2032-2045.

26. Sharif $A$, Hecking $M$, de Vries $A P$ et al. Proceedings from an international consensus meeting on posttransplantation diabetes mellitus: recommendations and future directions. Am J Transplant. 2014; 14 (9): 1992-2000.

27. Hiatt WR. Medical treatment of peripheral arterial disease and claudication. N Engl J Med. 2001; 344: 1608-1621.

28. Papanas N, Liakopoulos V, Maltezos E, Stefanidis I. The diabetic foot in end stage renal disease. Ren Fail. 2007; 29 (5): 519-528.

29. Ceresa CD, Aitken E, Dempster NJ, Kingsmore D. Outcomes of renal transplantation in patients with major lower limb amputation. Transplant Proc. 2014; 46 (1): 115-120.

30. Aitken E, Ramjug S, Buist L, Kingsmore D. The prognostic significance of iliac vessel calcification in renal transplantation. Transplant Proc. 2012; 44 (10): 2925-2931.

31. Baker RJ, Mark PB, Patel RK, Stevens KK, Palmer N. Renal association clinical practice guideline in post-operative care in the kidney transplant recipient. BMC Nephrol. 2017; 18 (1): 174.

32. Zelle DM, Corpeleijn E, Deinum $J$ et al. Pancreatic betacell dysfunction and risk of new-onset diabetes after kidney transplantation. Diabetes Care. 2013; 36: 1926-1932.

33. Kervinen MH, Lehto S, Helve J, Grönhagen-Riska C, Finne P. Type 2 diabetic patients on renal replacement therapy: probability to receive renal transplantation and survival after transplantation. PLoS One. 2018; 13 (8): e0201478.

34. Sharif A, Baboolal K. Complications associated with newonset diabetes after kidney transplantation. Nat Rev Nephrol. 2011; 8 (1): 34-42.

35. Shivaswamy V, Boerner B, Larsen J. Post-transplant diabetes mellitus: causes, treatment, and impact on outcomes. Endocr Rev. 2016; 37 (1): 37-61. doi: 10.1210/er.2015-1084.

36. Cai R, Wu M, Lin M, Guo X, Xing Y. Pretransplant homeostasis model assessment of insulin resistance and fasting plasma glucose predict new-onset diabetes after renal transplant in chinese patients. Transplant Proc. 2019; 51 (3): 768-773.

37. Morgan RD, O'Callaghan JM, Knight SR, Morris PJ. Alemtuzumab induction therapy in kidney transplantation: a systematic review and meta-analysis. Transplantation. 2012; 93 (12): 1179-1188.

38. Cole EH, Johnston O, Rose CL, Gill JS. Impact of acute rejection and new-onset diabetes on long-term transplant graft and patient survival. Clin J Am Soc Nephrol. 2008; 3 (3): 814-821.

39. Cooper L, Oz N, Fishman G et al. New onset diabetes after kidney transplantation is associated with increased mortality-A retrospective cohort study. Diabetes Metab Res Rev. 2017; 33 (8). doi: 10.1002/dmrr.2920.

40. Ghisdal L, Bouchta NB, Broeders N et al. Conversion from tacrolimus to cyclosporine A for new- onset diabetes after transplantation: a single-centre experience in renal transplanted patients and review of the literature. Transplant Int. 2008; 21: 146-151.

41. Sharif A, Shabir S, Chand S, Cockwell P, Ball S, Borrows R. Meta-analysis of calcineurin-inhibitor-sparing regimens in kidney transplantation. J Am Soc Nephrol. 2011; 22 (11): 2107-2718.

42. Johnston O, Rose CL, Webster AC, Gill JS. Sirolimus is associated with new-onset diabetes in kidney transplant recipients. J Am Soc Nephrol. 2008; 19: 1411-1418.

43. Kleinsteuber A, Halleck F, Khadzhynov D et al. Impact of preexisting comorbidities on long-term outcomes in kidney transplant recipients. Transplant Proc. 2018; 50 (10): 3232-3241.

44. Johal S, Jackson-Spence F, Gillott $\mathrm{H}$ et al. Pre-existing diabetes is a risk factor for increased rates of cellular rejection after kidney transplantation: an observational cohort study. Diabet Med. 2017; 34 (8): 1067-1073.

45. He X, Johnston A. Early acute rejection does not affect chronic allograft nephropathy and death censored graft failure. Transplant Proc. 2004; 36 (10): 2993-2996.

46. Woodle ES, First MR, Pirsch J, Shihab F et al. A prospective, randomized, double-blind, placebo-controlled multicenter trial comparing early ( 7 day) corticosteroid cessation versus longterm, low-dose corticosteroid therapy. Ann Surg. 2008; 248 (4): 564-77. doi: 10.1097/SLA.0b013e318187d1da.

47. Woodle E, Clark S, Stewart D et al. Fifteen year outcomes in the astellas double blind randomized controlled trial (RCT) comparing early corticosteroid withdrawal (CSWD) and longterm corticosteroid therapy continuation (CCS) in kidney transplantation [abstract]. Am J Transplant. 2019; 19 (suppl 3).

48. Pascual J, Galeano C, Royuela A, Zamora J. A systematic review on steroid withdrawal between 3 and 6 months after kidney transplantation. Transplantation. 2010; 90 (4): 343-349.

Correspondencia:

Dra. Itzel Anahí Martínez-Juárez

E-mail: draitzelmartinez@gmail.com 